\title{
Obtaining informed consent for anaesthesia in elective surgery at a tertiary-care hospital: practices and ethical-legal context

\author{
Casimiro L.G. ${ }^{1}$, Pereira S. ${ }^{2}$, Azevedo B. ${ }^{1}$, Pires S. ${ }^{3}$, Abelha F. ${ }^{1,2}$, Mourão J. ${ }^{1,2}$ \\ ${ }^{1}$ Centro Hospitalar São João E.P.E., Dept of Anaesthesiology, Porto, Portugal, \\ ${ }^{2}$ Faculty of Medicine, University of Porto, Dept of Anaesthesiology, Porto, Portugal \\ 3 Public Ministry
}

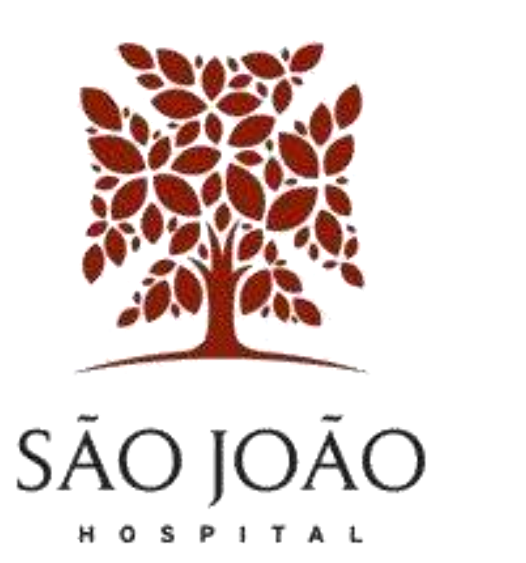

\section{BACKGROUND AND GOUL OF STUDY}

Currently there's a growing ethical and legal concern regarding the practice of informed consent (IC) for anaesthesia. This practice has not been adopted on a regular basis in all institutions, despite our general health institution recommending it. The anaesthetic practice has inherent risks, aside those from the surgery specific-ones, which makes the anaesthetic consent of great importance. Anaesthetic IC should never be neglected. The aim of this study was to evaluate the degree of implementation of specific informed consent for anaesthesia in the context of elective surgery at a tertiary hospital.

\section{MATERIALS AND METHODS}

It was conducted an observational prospective study.

\section{Inclusion criteria}

Patients aged $>60$ years old

Submitted to elective surgery

May-July 2017

Demographic data, the Charlson Comorbidity Score, previous medical history, clinical records and Clinical Frailty Score were collected. The patients were divided in two groups, those who had informed consent signed by the anaesthesiologist (IC) and those who had not (NIC). The Chi-square, Fisher's exact or Mann-Whitney $U$ tests were used for comparison.

\section{RESULTS AND DISCUSSION}

- It was obtained a sample of $\underline{230}$ patients.

- Only $41.7 \%$ of the informed consent were signed by the anaesthesiologist, while $97.8 \%$ were signed by the surgeon.

\begin{tabular}{|c|c|c|c|c|c|c|c|}
\hline Variable & IC & NIC & $P$ value & Variable & IC & NIC & $P$ value \\
\hline $\begin{array}{l}\text { Female } n(\%) \\
\text { Male } \mathrm{n}(\%)\end{array}$ & $\begin{array}{l}54(43.5) \\
42(39.6)\end{array}$ & $\begin{array}{l}70(56.5) \\
64(60.4)\end{array}$ & 0.547 & & $2(33.3)$ & $4(66.7)$ & 0.508 \\
\hline Age $n(p 25-p 75)$ & $69(65.0-77.0)$ & $70(65.0-77.0)$ & 0.863 & No & $94(42.0)$ & $130(58.0)$ & \\
\hline $\begin{array}{l}\text { ASA Score n (\%) } \\
1 \\
2\end{array}$ & $6(60.0)$ & $4(40.0)$ & 0.046 & $\begin{array}{l}\text { Psychiatric disease } \mathrm{n}(\%) \\
\text { Yes } \\
\text { No }\end{array}$ & $\begin{array}{l}15(45.5) \\
81(41.1)\end{array}$ & $\begin{array}{c}18(54.5) \\
116(58.9)\end{array}$ & 0.640 \\
\hline 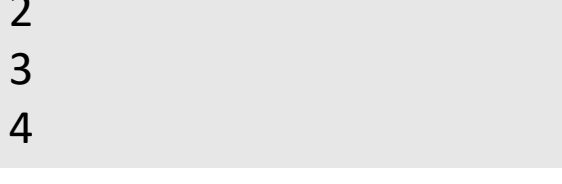 & $\begin{array}{l}56(44.8) \\
32(37.6) \\
2(20.0)\end{array}$ & $\begin{array}{l}69(55.2) \\
53(62.4) \\
8(80.0)\end{array}$ & & $\begin{array}{l}\text { Anemia n (\%) } \\
\text { Yes } \\
\text { No }\end{array}$ & $\begin{array}{l}13(27.7) \\
83(45.4)\end{array}$ & $\begin{array}{c}34(72.3) \\
100(54.6)\end{array}$ & 0.028 \\
\hline $\begin{array}{l}\text { Frailty Clinical Scale } \\
\text { Very Fit } \\
\text { Well } \\
\text { Managing Well }\end{array}$ & $\begin{array}{l}11(45.8) \\
21(45.7) \\
27(38.0)\end{array}$ & $\begin{array}{l}13(54.2) \\
25(54.3) \\
44(62.0)\end{array}$ & 0.844 & $\begin{array}{l}\text { Heart failure } n(\%) \\
\text { Yes } \\
\text { No }\end{array}$ & $\begin{array}{l}7(38.9) \\
89(42.0)\end{array}$ & $\begin{array}{c}11(61.1) \\
123(58.0)\end{array}$ & 0.798 \\
\hline $\begin{array}{l}\text { Vulnerable } \\
\text { Midly Frail } \\
\text { Moderately Frail }\end{array}$ & $\begin{array}{l}14(35.9) \\
9(56.2) \\
9(42.9)\end{array}$ & $\begin{array}{l}25(64.1) \\
7(43.8) \\
12(57.1)\end{array}$ & & $\begin{array}{l}\text { Isquemic heart disease } n(\%) \\
\text { Yes } \\
\text { No }\end{array}$ & $\begin{array}{c}8(42.1) \\
88(41.7)\end{array}$ & $\begin{array}{c}11(57.9) \\
123(58.3)\end{array}$ & 0.973 \\
\hline Severely Frail & $5(38.5)$ & $8(61.5)$ & & Miocardial infarction n (\%) & & & \\
\hline Charlson score $n(p 25-p 75)$ & $5.0(3.0-9.0)$ & $7.0(4.0-10.0)$ & 0.017 & $\begin{array}{l}\text { Yes } \\
\text { No }\end{array}$ & $\begin{array}{c}6(37.5) \\
90(42.1)\end{array}$ & $\begin{array}{c}10(62.5) \\
124(57.9)\end{array}$ & 0.721 \\
\hline $\begin{array}{l}\text { Yes } \\
\text { No }\end{array}$ & $\begin{array}{l}2(13.3) \\
94(43.7)\end{array}$ & $\begin{array}{c}13(86.7) \\
121(56.3)\end{array}$ & 0.021 & $\begin{array}{l}\text { Chronic obstructive } \\
\text { pulmonarydisease } n(\%) \\
\text { Yes } \\
\text { No }\end{array}$ & $\begin{array}{c}4(36.4) \\
92(42.0)\end{array}$ & $\begin{array}{c}7(63.6) \\
127(58.0)\end{array}$ & 0.484 \\
\hline
\end{tabular}

- It was identified a low practice of informed consent for anaesthesia procedures.

- There seems to be an association regarding an increased absence of informed anesthetic consent and:

- Higher ASA score and higher Charlson score.

- Previous history of stroke

- Anemia

- The informed consent is essential for the patient clarification about the anaesthesia practice as a unique medical speciality.

- It is a ethical e legal duty of great importance that cannot be neglected. 